\title{
A Comparative Three-Dimensional Finite Element Study of Two Space Regainers in the Mixed Dentition Stage
}

\author{
Mohamed Ahmed Abdel Hakim Melegy ${ }^{1}$ Nagwa Mohamed Ali Khatab ${ }^{2} \quad$ Kareem Maher Gaber Mohamed
} Ahmad Abdel Hamid Elheeny²

${ }^{1}$ Ministry of Health and Population, El Minia, Egypt

${ }^{2}$ Department of Paediatric and Community Dentistry, Faculty of Dentistry, Minia University, Minya, El Minia, Egypt

${ }^{3}$ Department of Orthodontic, Faculty of Dentistry, Minia University, Minya, El Minia, Egypt

Eur J Dent 2020;14:107-114

\begin{abstract}
Address for correspondence Ahmad Abdel Hamid Elheeny, PhD, Department of Paediatric and Community Dentistry, Faculty of Dentistry, Minia University Ard Shalaby, Postal code, 61519. Province, Minya, El Minia, Egypt (e-mail: ahmed.elheny@yahoo.com).
\end{abstract}

\begin{abstract}
Keywords

- mixed dentition

- finite element analysis

- stress distribution

Objectives This study aims to compare the stress distribution and displacement that resulted from the use of a Gerber space regainer and sagittal distalizer using three-dimensional finite element analysis.

Materials and Methods Three-dimensional simulated models of the appliances were developed using a software. The forces applied by the two appliances were $3 \mathrm{~N}$ (tipping) and $15 \mathrm{~N}$ (bodily), respectively. Displacement and von Mises stress on the compact and cancellous bone, periodontal ligament (PDL), crowns of the mandibular first, second permanent molars, and deciduous canines were calculated. Stress distribution and displacement values were measured via linear static analysis.

Results Gerber space regainer showed greater displacement than that produced by the sagittal distalizer at the first permanent molar. However, such displacement was less at the other tested points when compared with that delivered by sagittal distalizer. The stresses created by Gerber appliance were higher in the crown and PDL of the deciduous canine than the crown of the first permanent molar crown.

Conclusions Gerber appliance generates more distal force and less stress concentration on the crown of the mandibular first permanent molar than that created by the sagittal distalizer. On the other hand, stress concentrations produced by Gerber space regainer are found to be more on the crown and PDL of the deciduous canine. Therefore, it can be concluded that the use of Gerber appliance needs more anchorage.
\end{abstract}

\section{Introduction}

Premature loss of deciduous dentition has significant impacts on the growing child. ${ }^{1}$ These solicitudes are not only confined to functional, physiological, or esthetic disturbances but also can lead to the development of malocclusion and loss of guidance of erupting permanent teeth. ${ }^{1-3}$ The possible outcomes of early loss of primary teeth must be carefully assessed to determine the most suitable orthodontic intervention. ${ }^{4}$ There is a consensus that arch length discrepancies as a result of premature extraction of second deciduous molars are more obvious than that associated with early wastage of the first deciduous molars. ${ }^{4,5}$ Space problems are announced in terms of occupying the space left by the early loss of the mandibular second deciduous molars. ${ }^{5,6}$ Therefore, interceptive measures such as repositioning the mandibular first permanent molars using an appliance are necessary. ${ }^{7}$ A variety of appliances either removable, such as Hawley's appliances and C-space regainer, or fixed, such as sliding loop regainer, open coiled
DOI https://doi.org/ 10.1055/s-0040-1702254 ISSN 1305-7456.
License terms

()(1) $\Theta \circledast$ 
space regainer, lip bumper, distal jet appliance, and Gerber space regainer, have been utilized to accomplish such task. ${ }^{7}$

Gerber space regainer is a seat-side appliance that may be manufactured in a moderately brief span as it does not demand laboratory procedures. Nickel-titanium (Ni-Ti) coil springs are the main component. Open-coil springs convey a relatively more constant load value in the superelastic region; therefore, a more coveted persistent force can be acquired. ${ }^{8}$ Since it is a fixed appliance, patient acquiescence has to be good and oral hygiene ought to be preserved as the appliance was self-cleansing. However, the requirement for banding may influence gingival status and recurrent caries might develop under the band. The Ni-Ti coil springs were found to deliver light, continuous force of 250 to $350 \mathrm{~g}$ (0.025-0.034 MPa) with an average of $300 \mathrm{~g}(0.029 \mathrm{MPa}){ }^{8,9}$ A sagittal appliance is a removable appliance with a screw incorporated for the distalization of the first permanent molars. The anchorage is gained through the remaining teeth anterior to the first permanent molars. The removable nature of the appliance also makes it patient dependent and compliance effect efficiency. ${ }^{10}$ On the basis of assessment, the mechanical properties of different prosthetic designs and treatment planning concepts and precise reproduction of simulation of the functional differences between different supporting tissues are required. On the extent of structural engineering, the use of the finite element method (FEM) means to set up a condition of tension and deformation of an arbitrary-geometry solid submitted to external actions. ${ }^{11}$ Fine element analysis (FEA) is a numeric analytical method that permits the calculation of stresses and displacements. ${ }^{12}$ FEM is nearly similar to the clinical situation; however, the qualitative behavior of a dry skull doesn't mimic with a high level of exactness in the clinical circumstance. ${ }^{13}$ It is conceivable to demonstrate the way that the permanent first molar moves distally and study the commitment effect of molar distalization such as flaring of anterior teeth due to the loss of anchorage the force impact craniofacial structures and a profile change in the lower third of the face. ${ }^{14,15}$ FEM has several merits, such as (1) noninvasive technique; (2) theoretically, the definite value of applied stress at any point can be measured; (3) teeth and their supporting structures as well as the craniofacial bones can be simulated and mechanical properties of these structures can be assigned; (4) the applied force direction, magnitude, and application are easily modified to mimic the clinical situation, and (5) reproducibility does not affect the physical properties of relegated material. ${ }^{16-20}$ The aim of this investigation was to analyze and compare stress distribution as well as displacement of the dental and supporting structures after application of forces induced by Gerber space regainer and sagittal molar distalizer using FEA.

\section{Materials and Methods}

The current in-vitro study has been conducted in full accordance with the World Medical Association Declaration of Helsinki. The Ethics Committee of Faculty of Dentistry, Minia University, authorized the research conduction in Pediatric Dentistry and Orthodontic Departments, Faculty of
Dentistry, Minia University. An informed consent was signed by the father's child to use computed tomography (CT) images after clarifying the aim and the purpose of the research. The child undergoes orthodontic treatment. The inclusion criteria were unilateral localized sever space between more than $3 \mathrm{~mm}$ and less than $4 \mathrm{~mm} .^{21}$

Construction of the finite element (FE) model:

A flowchart of the FE process is illustrated in - Fig. 1.

\section{Getting the geometrical outline}

The FE model was constructed from CT photographs of an 8 -year-old child to simulate the child's mandible. A total of 60 CT slices saved as DICOM (Digital Imaging and Communications in Medicine) files were taken using Toshiba Aquilion 64 multi-slice scanner $(120 \mathrm{KV}, 150 \mathrm{~mA}$, with a resolution of $512 \times 512$ matrix and a slice thickness of $0.5 \mathrm{~mm}$ ). A Materialize Interactive Medical Image Control System (MIMICS), 10.01 for Intel x86 platform v10.2.1.2 (Materialise NV, Belgium), was applied to extract a three-dimensional (3D) model from the CT images.

\section{Model components 3D drawing}

The model was imported into Solidworks 2017 sp0.0 software (Solidworks Corporation, France) to design a tetrahedral FE mesh; the mandible incorporating teeth and alveolar bone had meshed into $1 \mathrm{~mm}$ tetrahedrons. The teeth, alveolar bone, and periodontal ligament (PDL) were viewed as homogenous and isotropic. A small sliding condition and the Lagrange multiplier method were utilized to characterize the contact interface. The cortical and spongy bone, teeth, stainless steel (SS) wire, and acrylic resin are dense. Material properties of the components of the dental follicle encompassing the unerupted molar were similar to those of the PDL that had a lower density (- Table 1). ${ }^{22,23}$ The contacts between the teeth were postulated to be frictionless. The thickness of the PDL and cortical bone was considered to be 0.2 and $3.4 \mathrm{~mm}$, respectively. ${ }^{24}$ Mating was done between teeth, PDL, and the mandible with the reported angulations and inclinations to achieve model idealization and standardization ${ }^{25}$ as illustrated in - Table 2.

\section{Appliances design and activation}

\subsection{Gerber space regainer}

The space regainer was fixed in the permanent first molar by a cemented tubed band. SS bar ( $1.0 \mathrm{~mm}$ in diameter) was connected to the bands through the tube, extended anteriorly till reaching the distolabial surface of deciduous canine, then bent lingually around the deciduous canine and finally returned back posteriorly, till reaching the lingual tube. Mating was done by assembling the mesial opening of the tube and the arm distal free end of the arm, and then moving the SS arm into the distal end of the tube by dragging option. Coil spring is the source of force used in space gaining and is represented by a force arm that applied at all device force points (-Fig. 2A). The tipping force was delivered after open coil spring activation was $3 \mathrm{~N}$ and directed horizontally through the long axis of the SS rod in both directions to avoid any 

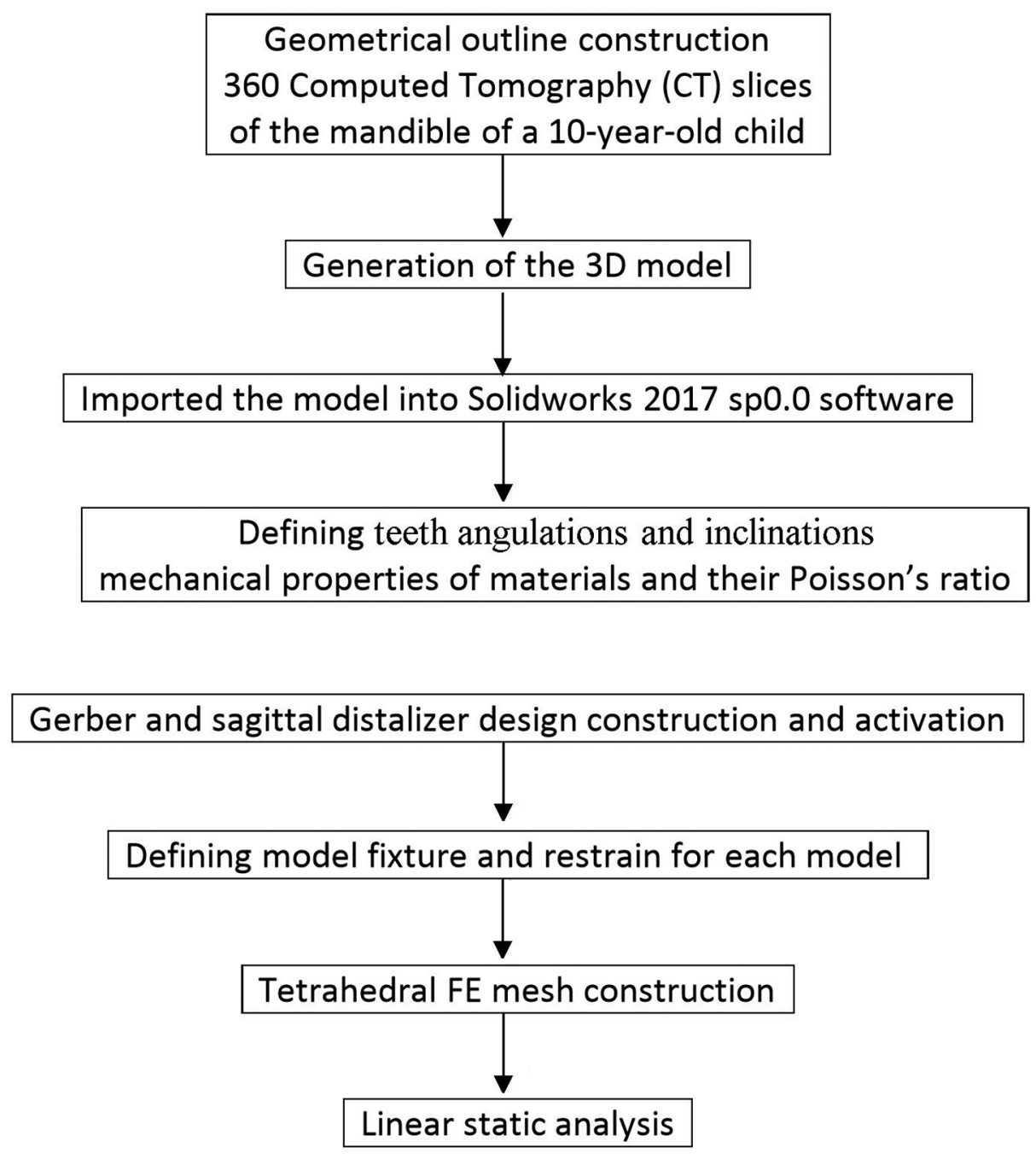

Fig. 1 Flowchart of finite element method procedures.

Table 1 Material property data and their Poisson's ratio

\begin{tabular}{|l|l|l|}
\hline Material & $\begin{array}{l}\text { Young's modulus } \\
(\mathrm{MPa})\end{array}$ & Poisson's ratio \\
\hline Cortical bone & $1.37 \times 10^{4}$ & 0.30 \\
\hline Cancellous bone & $7.90 \times 10^{3}$ & 0.30 \\
\hline Acrylic & $2.30 \times 10^{4}$ & 0.40 \\
\hline Tooth & $2.07 \times 10^{4}$ & 0.30 \\
\hline Stainless steel wire & $2.00 \times 10^{5}$ & 0.30 \\
\hline Periodontal ligament & 50.00 & 0.49 \\
\hline
\end{tabular}

eccentric force or dissipate any force away from the application point. ${ }^{25}$

\subsection{Sagittal molar distalizer}

An acrylic body of $3 \mathrm{~mm}$ thickness was considered the major component with its superior border, followed the gingival scalloping, the inferior border extended to the vestibule, and the distal surface extended to the mandibular second molar. Splitting in the acrylic body was performed in the
Table 2 Teeth angulations and inclinations

\begin{tabular}{|l|l|l|}
\hline Tooth & Angulations & Inclinations \\
\hline $\begin{array}{l}\text { Central permanent } \\
\text { incisor }\end{array}$ & $1^{\circ} \pm 0.5$ & $-6.65^{\circ} \pm 0.6$ \\
\hline $\begin{array}{l}\text { Lateral permanent } \\
\text { incisor }\end{array}$ & $1^{\circ} \pm 0.3$ & $-6.48^{\circ} \pm 0.5$ \\
\hline Deciduous canine & $3^{\circ} \pm 0.6$ & $-6.79^{\circ} \pm 0.5$ \\
\hline $\begin{array}{l}\text { First permanent } \\
\text { molar }\end{array}$ & $2^{\circ} \pm 0.5$ & $-20.59^{\circ} \pm 0.5$ \\
\hline $\begin{array}{l}\text { Second permanent } \\
\text { molar }\end{array}$ & $2^{\circ} \pm 0.5$ & $-10.5^{\circ} \pm 0.5$ \\
\hline
\end{tabular}

middle of the edentulous space. Anchorage clasps connected the acrylic body at the area of the first permanent molars and anterior segment. Mating the free end of the wire and superior border of the acrylic body, the SS wire was moved to the inside of acrylic body by dragging option. The jackscrew was the source of force used in distalization and it was represented by force arm applied in all device force points (-Fig. 1B). The bodily movement forces of $15 \mathrm{~N}$ were applied by activation of the screw and directed horizontally through 


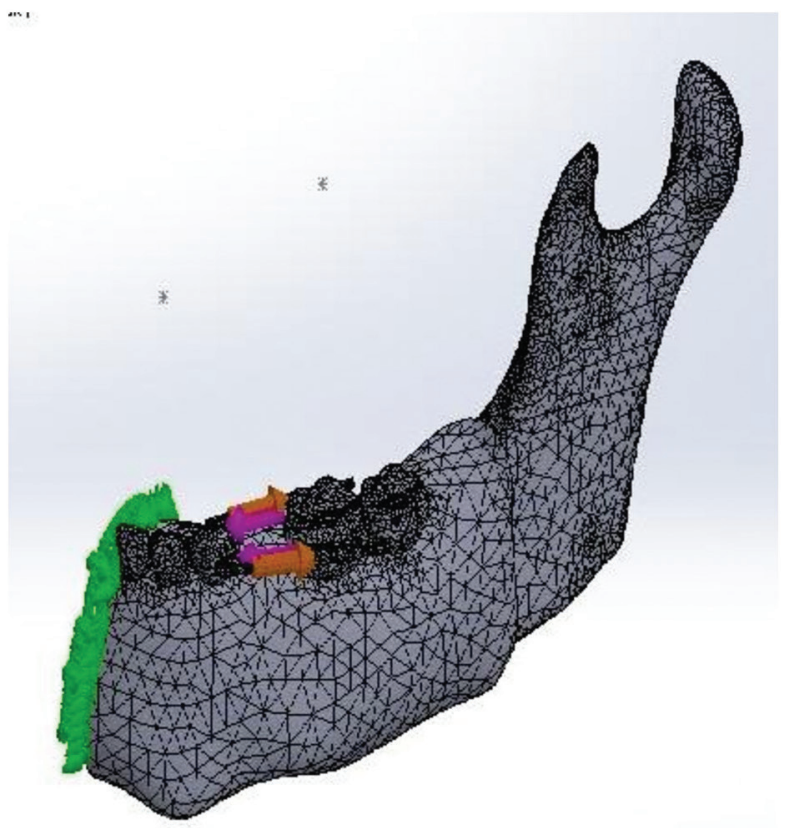

(A)

Fig. 2 Gerber space regainer (A) and sagittal molar distalizer (B).

the long axis of the force arm in both directions to avoid any eccentric force or dissipate any force away from the application point $t^{9,25}$

\section{Material characteristics}

The cortical and spongy bone, teeth, SS wire, and acrylic resin were dense. The material properties of the dental follicle elements around the unerupted molar were similar to those of the lower density PDL. ${ }^{26}$

\section{Defining model fixture and restrain for each model}

For both appliances, all the elements were allowed to translate in all directions except rotation. The only restraint applied was a fixed restraint on the condylar neck. ${ }^{27}$

\section{Meshing}

A high-quality solid mesh was utilized in this investigation to produce 3D parabolic tetrahedral solid elements. As a solid mesh was permitted to interpret on any of the three orthogonal bearings unless a limitation was connected, in any case, no rotation was allowed. ${ }^{27}$ The number of nodes for Gerber appliance was 1,29,515 and 76,463 solid elements. The FE model of sagittal distalizer consisted of $1,51,945$ nodes and $1,40,512$ solid elements.

\section{Analysis}

Linear static analysis was performed via Solidworks 2017 sp0.0.

After analysis, a result tree was activated. Both displacement and von Mises stress on the compact and spongy bone, PDL and teeth crowns of the deciduous canine, and the first and second molars were calculated.

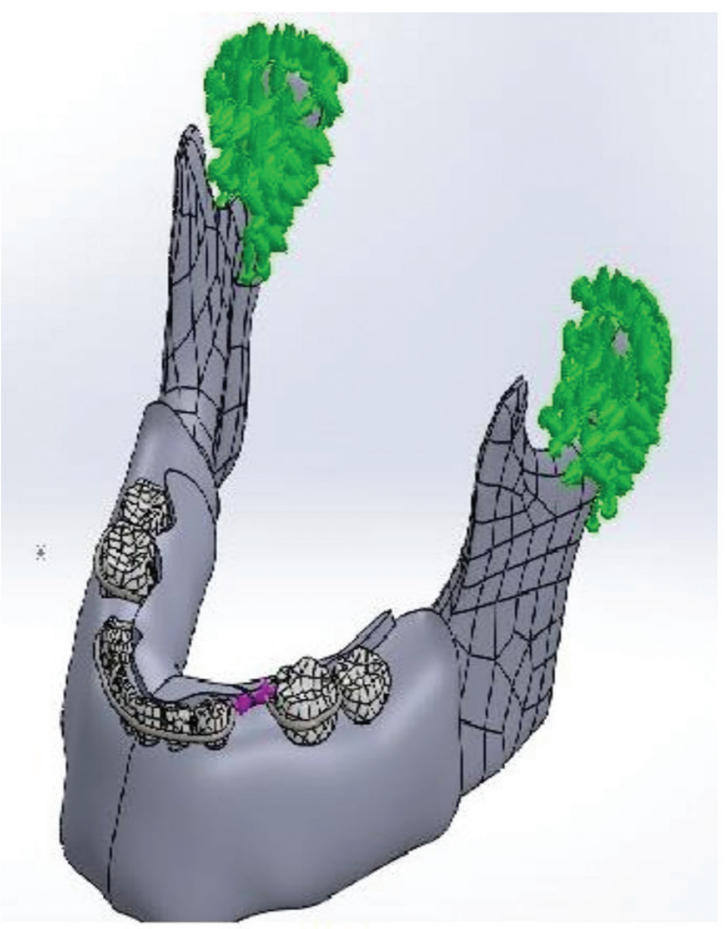

(B)

\section{Results}

\section{Displacement}

The maximum displacement of the first permanent molar was generated by Gerber regainer $(8.91 \mu \mathrm{m})$, while sagittal distalizer produced a higher displacement at the area of six anterior teeth (i.e., anchorage segment) $(7.061 \mu \mathrm{m})$. The displacement values created by Gerber appliance at the compact and spongy bone ( 2.710 and $2.702 \mu \mathrm{m}$, respectively) as well as the PDL of deciduous canine $(2.44 \mu \mathrm{m})$, first and second permanent molars (5.907 and $1.924 \mu \mathrm{m}$, respectively) were less than that created by the sagittal distalizer appliance (1.51 $\mu \mathrm{m}$ for PDL, $6.021 \mu \mathrm{m}$ for the first permanent molar, and $4.015 \mu \mathrm{m}$ for the second permanent molar) (-Table 3, -Figs. 3 and 4 ). Gerber appliance produced distal tipping movement, while the movement type generated by the sagittal distalizer was bodily.

\section{Stress Distribution}

The von Mises stress distribution demonstrated the highest stress concentrations at the crown of deciduous canine in Gerber regainer (11.2 MPa), which was much higher than that recorded by the sagittal appliance (5.12 MPa). However, stresses delivered by sagittal distalizer on the crown of the first permanent molar $(0.69 \mathrm{MPa})$ were slightly higher than stresses delivered by Gerber space regainer (0.4 MPa). The spongy bone $(0.045 \mathrm{MPa})$, the PDL of the deciduous canine $(0.065 \mathrm{MPa})$, and both first and second permanent molars (0.151 and $0.094 \mathrm{MPa}$, respectively) recorded higher stress concentrations with Gerber appliance than that recorded with the distalizer device (-Table 2 ). The compact bone showed high-stress concentration in sagittal distalizer of 
Table 3 Stress distribution and displacement values

\begin{tabular}{|l|l|l|l|l|}
\hline \multirow{2}{*}{ Point } & \multicolumn{2}{|c|}{ Stress (Mpa) } & \multicolumn{2}{c|}{ Displacement $(\mu \mathrm{m})$} \\
\cline { 2 - 5 } & Gerber regainer & Sagittal distalizer & Gerber regainer & Sagittal distalizer \\
\hline Compact bone & 0.2119 & 0.334 & 2.710 & 6.010 \\
\hline Spongy bone & 0.045 & 0.027 & 2.702 & 6.002 \\
\hline Deciduous canine crown & 11.20 & 5.126 & 6.206 & 7.061 \\
\hline First molar crown & 0.40 & 0.696 & 8.910 & 6.102 \\
\hline Second molar crown & 0.015 & 0.224 & 1.910 & 4.010 \\
\hline PDL of deciduous canine & 0.065 & 0.032 & 2.440 & 1.510 \\
\hline PDL of first permanent molar & 0.151 & 0.085 & 5.907 & 6.021 \\
\hline PDL of second permanent molar & 0.094 & 0.025 & 1.924 & 4.015 \\
\hline
\end{tabular}

Abbreviation: PDL, periodontal ligament.
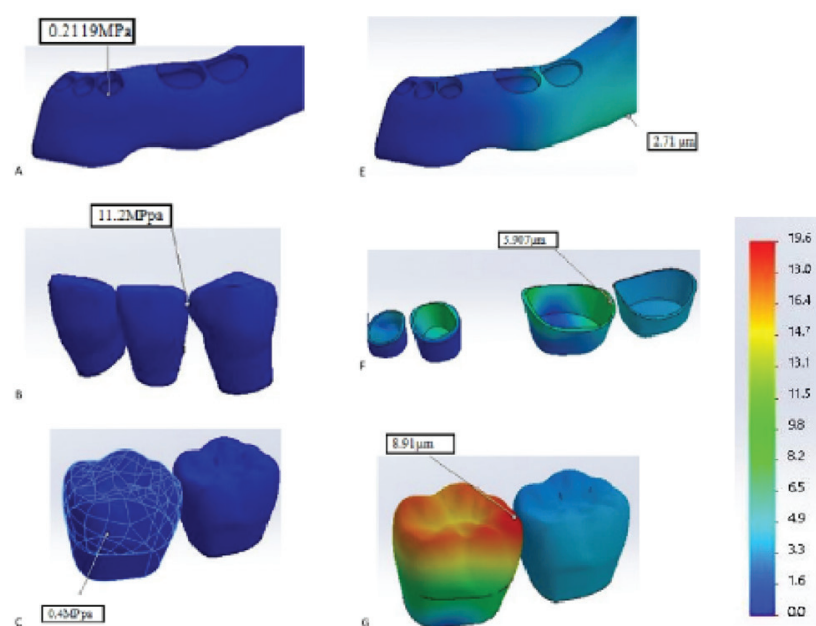

c. $0.40 \mathrm{pa}$
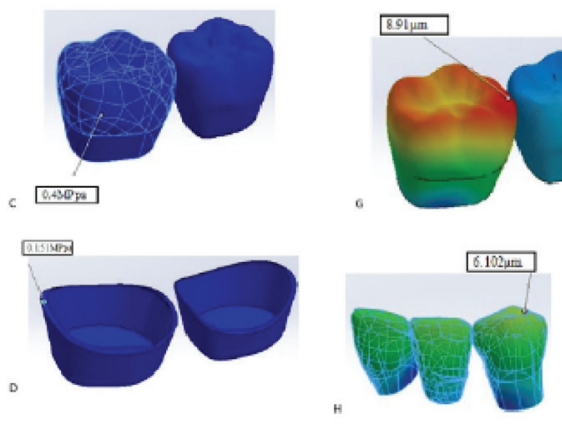

Fig. 3 Stress distribution of Gerber space regainer on the compact bone ( $\mathbf{A}$ and $\mathbf{E})$, deciduous canine ( $\mathbf{B}$ and $\mathbf{F})$, first permanent molar ( $\mathbf{C}$ and $\mathbf{G})$, and periodontal ligament ( $\mathbf{D}$ and $\mathbf{H})$.

$0.334 \mathrm{MPa}$ in comparison with the other device (0.212 MPa). Moreover, stresses generated by sagittal distalizer, maximum stress recorded at the area of six anterior teeth that represented the anchorage anterior segment (25.80 MPa), with the highest stress at the deciduous canine (5.13 MPa). Gerber regainer generated maximum stress of $19.60 \mathrm{MPa}$ at the contact between coil spring and the band with the maximum stress at the deciduous canine (11.20 MPa).

\section{Discussion}

In the current study, a mixed dentition mandible with an approximate age of 10 years was scanned to reconstruct a 3D FE model. The point of force application, magnitude, and force direction with all the two devices was simulated as per the clinical situation. The stress distribution and deformation
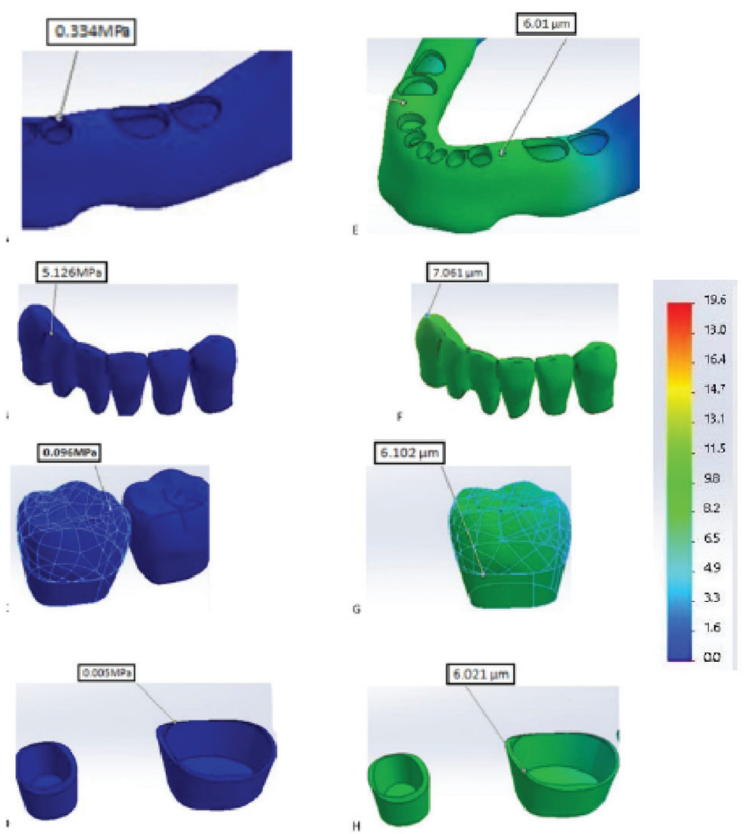

Fig. 4 Stress distribution and displacement of sagittal molar distalizer in the compact bone ( $\mathbf{A}$ and $\mathbf{E}$ ), deciduous canine ( $\mathbf{B}$ and $\mathbf{F}$ ), first permanent molar ( $\mathbf{C}$ and $\mathbf{G}$ ), and periodontal ligament ( $\mathbf{D}$ and $\mathbf{H}$ ).

influence the dental and dentoalveolar structures were measured, analyzed, and compared with other previously available data. At whatever point, when a load is applied to a structure, deformation and stresses are generated, and this cannot be measured straightforwardly. In complex structures such as the stomatognathic system, FEA is the most advanced and reliable method.

In regard to the available data, the use of FEM in studying stress analysis of space regainer in pediatric dentistry was limited. Most of the available data used the FEA method does not study these two appliances for space regaining. Therefore, the differences between stress and displacement distribution pattern in our findings and other studies might be related to the difference in lines of action, direction, magnitude of the force, anchorage type, and the used model. However, in comparison with other studies in terms of the type of tooth 
movement, force-generating parts, effects in PDL, teeth, and bone would be useful. ${ }^{17,28-30}$

In regard to stresses generated by sagittal distalizer, maximum stress recorded at the area of the anchorage anterior segment, with the highest stress at the deciduous canine. However, Gerber regainer generated the maximum stress at the contact between the coil spring and the band with the maximum stress at the deciduous canine. These differences between the two appliances were attributed to lack of anchorage in Gerber regainer as the device anchored mainly on the first molar and deciduous canine only. These findings were in line with the results of Shetty et al, who reported high-stress concentration, resulting from the use of jackscrew in molar distalization, to be $7.78 \mathrm{~N}$ at the anchorage anterior segment. ${ }^{30}$ Erverdi et al and Sung et al recommended the use of a Nance appliance or lingual arch to overcome the anchorage deficiency during molar distalization using coil spring appliances. ${ }^{31,32}$ Also, Işeri et al reported high-stress levels at the maxillary canine and molar area but with minimal distractive effect due to high anchorage used..$^{17}$ Kang et al demonstrated high concentrations of stress around the miniscrews (an extra anchorage) of the modified palatal anchorage plate and bone-anchored pendulum apparatuses, and around the headgear bands of maxillary first molars. ${ }^{33}$ Moreover, Lee et al recorded the highest stress concentration that was found around the microimplants in rapid maxillary expander and conventional Hyrax arms. ${ }^{29}$ These findings were also in agreement with that reported by the current study about the maximum stress concentration around the anchorage area.

Gerber and sagittal appliances demonstrated minimal stress on the first permanent molar (0.4 and 0.6 MPa, respectively). This finding was compatible with the results of Kang et al who reported that the lowest stress distribution was at the first molar radicular apical third with the headgear than bone-anchored pendulum.

In regard to stresses delivered by the two appliances on the compact bone, stresses delivered by sagittal distalizer at the lingual alveolar crest of the deciduous canine were greater than that produced by Gerber regainer at the mesial alveolar crest of the erupting second molar. These findings were in accordance with the study performed by Lee et al, who reported high-stress concentration around the anchorage points. This finding explained our result where the lingual alveolar crest of the deciduous canine was close to the anterior anchorage in the sagittal distalizer. ${ }^{29}$

The PDL showed minimal stress concentration among all dental and supporting structures. Gerber regainer delivered $0.15 \mathrm{MPa}$ at the first molar, $0.09 \mathrm{MPa}$ at the second molar, and $0.06 \mathrm{MPa}$ at the deciduous canine. However, the sagittal distalizer delivered $0.09 \mathrm{MPa}$ at the first permanent molar and $0.03 \mathrm{MPa}$ at both the second molar and the deciduous canine. These upshots were fit with the outcomes of Feizbakhsh et al, who investigated the stress distribution at maxillary first molar periodontium employing straight-pull headgear with vertical and horizontal tubes and reported that the lowest stress concentration found at the PDL $(0.10 \mathrm{MPa}){ }^{28}$
Displacement results of the sagittal distalizer revealed a notable movement of posterior teeth due to force application near their center of resistance. However, tipping movements were more eminent in the direction of posterior teeth due to faraway force application from the center of resistance in Gerber regainer. These findings confirmed with preceding studies of Sung et al and Park et al who recorded that the whole arch displacement may be determined by the direct relationship between the entire arch center of resistance, force application point, and its vector. $^{32,34}$

Comparing displacement pattern stresses delivered by the two appliances on the compact bone, sagittal distalizer produced $6.01 \mu \mathrm{m}$ at the lingual alveolar crest of the deciduous canine, and Gerber regainer produced $2.710 \mu \mathrm{m}$ at the distal alveolar crest of the erupting second molar. These findings were confirmed by Kang et al and Park et al who reported the displacement distribution level at the compact bone with the headgear lower than the bone-anchored pendulum. ${ }^{22,34}$ This was in concurrence with the distal tipping movement that happened with Gerber application, and the bodily movement with the other appliance, and also Lee et al who reported that the high-stress concentration and displacement were obvious around anchorage points. ${ }^{29}$

Higher displacement was reported at the PDL of the first molar when compared to other dentition. Displacement was nearly equal in the both devices; $5.907 \mu \mathrm{m}$ for Gerber regainer and $6.021 \mu \mathrm{m}$ for sagittal distalizer. These findings were in agreement with the previous studies of Fongsamootr and Suttakul, about the effect of PDL on stress distribution and displacement of tooth and bone structure using FE simulation ${ }^{35}$ and by Feizbakhsh et al, who reported that the mechanical properties of PDL that were well flexibility caused the tooth to move independently within soft volume supported. The PDL allows tooth movement so that the high displacement value in PDL considered an indication of the efficacy of the used appliance. ${ }^{28}$

The limitations of the current study were attributed to the disadvantages of the linear FEA method that could be summarized as follows: (1) FEM models did not include the whole skull so that the results represented only the response of part of the craniofacial structure; (2) elements representing tissues were considered isotropic; (3) it did not consider the change in soft tissue material properties during growth as modules of elasticity and Poisson's ratio; (4) it did not consider in detail the residual stresses at the end of each jackscrew and coil spring activation; (5) the current study performed in linear static manner without the concern of time factor and the dynamic properties of the appliance. Finally, FEM was not the individual variability in the physiologic and anatomic structure which influenced the loading of these devices. On the other hand, although this study was a one-time study on a single mandible at one-time activation of both devices, the results not only give a detailed insight into the initial mechanical response of the biological tissues of dental and dentoalveolar region to distalization therapy but also help understand and predict the compounded effects with subsequent activations. 


\section{Conclusions}

Gerber and sagittal distalizer appliances are capable of regaining the space lost by the mesial movement of the mandibular first permanent molar. However, the resultant stress concentration and displacement produced by the two appliances are different. Gerber appliance generates more distal force and less stress concentration on the crown of the mandibular first permanent molar than that created by the sagittal distalizer. On the other hand, stress concentrations produced by Gerber space regainer are found to be more on the crown and PDL of the deciduous canine. Therefore, it can be concluded that the use of Gerber appliance needs more anchorage. In addition, the sagittal distalizer appliance has an advantage over Gerber appliance as it produces slow and distal bodily movement to regain space in children. Further studies using nonlinear analysis can be beneficial.

\section{Funding}

None.

\section{Study Significance}

- Premature loss of deciduous dentition is common and has significant impacts on the growing child including a decrease in the arch perimeter. Therefore, the space regaining is mandatory.

- This study simulates two space regainer appliances using a FEM that simulates the clinical situation providing dependable data in a noninvasive way about stress distribution and displacement values in the dentoalveolar structures in mixed dentition stage.

- Limited data are available in such an era of pediatric dentistry, so comparing stress concentration and displacement simulation created by two space regainers with different types of tooth movements is viable in the dentist decision and treatment plan for space regaining.

\section{Conflict of Interest}

None declared.

\section{References}

1 Klapper BJ, Strizak-Sherwin R. Esthetic anterior space maintenance. Pediatr Dent 1983;5(2):121-123

2 Barbería E, Lucavechi T, Cárdenas D, Maroto M. Free-end space maintainers: design, utilization and advantages. J Clin Pediatr Dent 2006;31(1):5-8

3 Setia V, Pandit IK, Srivastava N, Gugnani N, Sekhon HK. Space maintainers in dentistry: past to present. J Clin Diagn Res 2013;7(10):2402-2405

4 Law CS. Management of premature primary tooth loss in the child patient. J Calif Dent Assoc 2013;41(8):612-618

5 Rock WP; British Society of Paediatric Dentistry. UK National Clinical Guidelines in Paediatric Dentistry. Extraction of primary teeth - balance and compensation. Int J Paediatr Dent 2002;12(2):151-153

6 Magnússon TE. The effect of premature loss of deciduous teeth on the spacing of the permanent dentition. Eur J Orthod 1979;1(4):243-249

7 Chalakkal P, Thomas AM, Akkara F, Pavaskar R. New design space regainers: 'lingual arch crossbow' and 'double banded space regainer' J Indian Soc Pedod Prev Dent 2012;30(2):161-165
8 Vibhute PJ. Open-coil retraction spring. Case Rep Dent 2011;2011:435709

9 von Fraunhofer JA, Bonds PW, Johnson BE. Force generation by orthodontic coil springs. Angle Orthod 1993;63(2):145-148

10 Singh G. Textbook of Orthodontics. New Delhi: JP Medical Ltd; 2015

11 Knop L, Gandini LG Jr, Shintcovsk RL, Gandini MREAS. Scientific use of the finite element method in orthodontics. Dental Press J Orthod 2015;20(2):119-125

12 Singh JR, Kambalyal P, Jain M, Khandelwal P. Revolution in Orthodontics: finite element analysis. J Int Soc Prev Community Dent 2016;6(2):110-114

13 Kumar A, Ghafoor H, Khanam A. A comparison of three-dimensional stress distribution and displacement of naso-maxillary complex on application of forces using quad-helix and nickel titanium palatal expander 2 (NPE2): a FEM study. Prog Orthod 2016;17(1):17

14 Yu J, Park JH, Bayome M, et al. Treatment effects of mandibular total arch distalization using a ramal plate. Korean J Orthod 2016;46(4):212-219

15 Kim YB, Bayome M, Park JH, et al. Displacement of mandibular dentition during total arch distalization according to locations and types of TSADs: 3D finite element analysis. Orthod Craniofac Res 2019;22(1):46-52

16 Ebadian B, Mosharraf R, Khodaeian N. Effect of cantilever length on stress distribution around implants in mandibular overdentures supported by two and three implants. Eur J Dent 2016;10(3):333-340

17 Işeri H, Tekkaya AE, Oztan O, Bilgiç S. Biomechanical effects of rapid maxillary expansion on the craniofacial skeleton, studied by the finite element method. Eur J Orthod 1998;20(4):347-356

18 Jafari A, Shetty KS, Kumar M. Study of stress distribution and displacement of various craniofacial structures following application of transverse orthopedic forces-a three-dimensional FEM study. Angle Orthod 2003;73(1):12-20

19 Kamble RH, Lohkare S, Hararey PV, Mundada RD. Stress distribution pattern in a root of maxillary central incisor having various root morphologies: a finite element study. Angle Orthod 2012;82(5):799-805

20 Singh SV, Bhat M, Gupta S, Sharma D, Satija H, Sharma S. Stress distribution of endodontically treated teeth with titanium alloy post and carbon fiber post with different alveolar bone height: a three-dimensional finite element analysis. Eur J Dent 2015;9(3):428-432

21 Proffit WR, Fields HW Jr, Sarver DM. Contemporary Orthodontics Massachusetts: Elsevier Health Sciences; 2006

22 Kang JM, Park JH, Bayome M, et al. A three-dimensional finite element analysis of molar distalization with a palatal plate, pendulum, and headgear according to molar eruption stage. Korean J Orthod 2016;46(5):290-300

23 Nalbantgil D, Tozlu M, Ozdemir F, Oztoprak MO, Arun T. FEM analysis of a new miniplate: stress distribution on the plate, screws and the bone. Eur J Dent 2012;6(1):9-15

24 Farnsworth D, Rossouw PE, Ceen RF, Buschang PH. Cortical bone thickness at common miniscrew implant placement sites. Am J Orthod Dentofacial Orthop 2011;139(4):495-503

25 Wichelhaus A, Geserick M, Ball J. A new nickel titanium rapid maxillary expansion screw. J Clin Orthod 2004;38(12): 677-680, quiz 671-672

26 Yu HS, Baik HS, Sung SJ, Kim KD, Cho YS. Three-dimensional finite-element analysis of maxillary protraction with and without rapid palatal expansion. Eur J Orthod 2007;29 (2): $118-125$

27 Nudehi S, Steffen J. Analysis of machine elements using SOLIDWORKS simulation 2018. USA, SDC Publications; 2018

28 Feizbakhsh M, Kadkhodaei M, Zandian D, Hosseinpour Z. Stress distribution in maxillary first molar periodontium using 
straight pull headgear with vertical and horizontal tubes: a finite element analysis. Dent Res J (Isfahan) 2017;14(2):117-124

29 Lee SC, Park JH, Bayome M, Kim KB, Araujo EA, Kook YA. Effect of bone-borne rapid maxillary expanders with and without surgical assistance on the craniofacial structures using finite element analysis. Am J Orthod Dentofacial Orthop 2014;145(5):638-648

30 Shetty P, Hegde AM, Rai K. Study of stress distribution and displacement of the maxillary complex following application of forces using jackscrew and nitanium palatal expander 2-a finite element study. J Clin Pediatr Dent 2009;34(1):87-93

31 Erverdi N, Koyutürk O, Küçükkeles N. Nickel-titanium coil springs and repelling magnets: a comparison of two different intra-oral molar distalization techniques. Br J Orthod 1997;24(1):47-53
32 Sung EH, Kim SJ, Chun YS, Park YC, Yu HS, Lee KJ. Distalization pattern of whole maxillary dentition according to force application points. Korean J Orthod 2015;45(1):20-28

33 Kang YG, Kim JY, Lee YJ, Chung KR, Park YG. Stability of miniscrews invading the dental roots and their impact on the paradental tissues in beagles. Angle Orthod 2009;79(2):248-255

34 Park M, Na Y, Park M, Ahn J. Biomechanical analysis of distalization of mandibular molars by placing a mini-plate: a finite element study. Korean J Orthod 2017;47(5):289-297

35 Fongsamootr T, Suttakul P. Effect of periodontal ligament on stress distribution and displacement of tooth and bone structure using finite element simulation. Eng J (NY) 2015;19(2):99-108 